\title{
Autologous EGFRt/BCMA-41BBz-targeted CAR T Cells
}

National Cancer Institute

\section{Source}

National Cancer Institute. Autologous EGFRt/BCMA-41BBz-targeted CART Cells. NCI

Thesaurus. Code C148168.

A preparation of autologous T-lymphocytes transduced with a retroviral vector expressing a chimeric antigen receptor (CAR) specific for the tumor-associated antigen (TAA) human B-cell maturation antigen (BCMA; tumor necrosis factor receptor superfamily member 17; TNFRSF17) fused to the co-stimulatory domain of 4-1BB (CD137) and the CD3-zeta (CD3z) T-cell signaling domain, and a truncated form of the human epidermal growth factor receptor (EGFRt), with potential immunostimulating and antineoplastic activities. Upon intravenous administration, autologous EGFRt/BCMA41BBz-targeted CAR T cells are directed to, and induce selective toxicity in, BCMAexpressing tumor cells. Devoid of both ligand-binding domains and tyrosine kinase activity, the expressed EGFRt facilitates in vivo detection of the administered, transduced T-cells and, if the administered T-cells cause unacceptable side effects, can promote elimination of those cells through a cetuximab-induced antibody-dependent cellular cytotoxicity (ADCC) response. The 4-1BB costimulatory signaling domain enhances both proliferation of T-cells and antitumor activity. BCMA, a receptor for both a proliferationinducing ligand (APRIL) and B-cell activating factor (BAFF), is a member of the tumor necrosis factor receptor superfamily (TNFRSF) and plays a key role in plasma cell survival. BCMA is found on the surfaces of plasma cells and is overexpressed on malignant plasma cells. 